\title{
Analytical approach to the design of microring resonators for nonlinear four-wave mixing applications
}

\author{
T. Hansson, * D. Modotto, and S. Wabnitz \\ Dipartimento di Ingegneria dell'Informazione, Università di Brescia, via Branze 38, 25123 Brescia, Italy \\ *Corresponding author: tobias.hansson@ing.unibs.it
}

Received January 23, 2014; revised March 23, 2014; accepted March 23, 2014; posted March 24, 2014 (Doc. ID 205306); published April 18, 2014

\begin{abstract}
An analytical approach for obtaining linear and nonlinear design parameters of microresonators is presented. The eigenmode/eigenfrequency problem of planar resonators is considered in detail, with an analytical closed-form approximation derived for resonators possessing a large radius to width ratio. The analysis permits the resonant frequencies and mode profiles to be determined together with the dispersion properties. The dependence of the effective nonlinear Kerr coefficient on the mode volume is further considered, and also the waveguide coupling together with estimates of the $Q$-value. Examples, which are in good agreement with numerical simulations, are presented for silicon resonators. The approach can be used for designing planar microring resonators for nonlinear four-wave mixing applications, such as optical Kerr frequency comb generation. (c) 2014 Optical Society of
\end{abstract} America

OCIS codes: (230.5750) Resonators; (230.7370) Waveguides; (230.7405) Wavelength conversion devices;

(230.7408) Wavelength filtering devices.

http://dx.doi.org/10.1364/JOSAB.31.001109

\section{INTRODUCTION}

Microring resonators show potential for revolutionizing the generation of Kerr frequency combs [1-3] . Optical frequency combs have a number of current and emerging applications for which they are attractive. These include, e.g., optical clocks, spectroscopy, gas sensing, arbitrary waveform generation, and precision frequency metrology $[4,5]$. Some of the most common types of resonators are planar [ $\underline{6}-\underline{8}]$, toroidal or spherical crystalline [9-11]. They have in common that they rely on the parametric four-wave mixing (FWM) $[\underline{1,12}]$ process for the nonlinear generation of frequency components from a continuous wave (CW) pump source. Resonators confine the light within a small volume and recirculate it within the cavity, which enhances the intensity and lowers the threshold for multi-wave mixing processes. Planar resonators are particularly interesting for application purposes since they have a small footprint and can be integrated on a chip. Planar resonators can also be folded to have virtually any shape, although they usually take the form of either rings or racetracks $[13,14]$. Different material platforms are additionally possible, depending on the operating range of wavelengths. Silica can, e.g., be used for telecommunication wavelengths while other materials, such as silicon, can be used for longer wavelengths in the near- and mid-infrared. However, it can be challenging to extend resonator technology from the telecommunication range to new wavelength regimes [15]. Most frequency combs to date have consequently been demonstrated in the nearinfrared using, e.g., crystalline whispering-gallery-mode resonators. These have the advantage over planar resonators of having very high $Q$-factors (e.g., $Q \approx 10^{9}$ ).

To design either a ring, racetrack, or other type of planar resonator for nonlinear applications, such as frequency comb generation, it is necessary to tailor the cavity dispersion properties. For soft excitation of a frequency comb utilizing modulation instability of the CW pump mode, one should preferably be in the anomalous dispersion regime. Soft comb excitations are also possible in the normal dispersion regime, but require both a large dispersion and detuning [16]. The dispersion profile can be controlled by suitably designing the resonator cross section such that the sum of material and geometric dispersions has the desired properties. It is furthermore important to account for the magnitude of nonlinear and coupling parameters. These can be determined using knowledge of the resonator eigenmodes. The strength of the nonlinearity depends on the effective nonlinear Kerr coefficient, which is inversely related to the mode volume. Although the mode volume, or the related mode area, is usually assumed to be constant, it does have a frequency dependence, which may also be important to consider for ultrabroadband frequency combs, such as octave spanning combs.

In this article, we apply an analytical approach to planar microring resonators. The width of the ring waveguide is an important design parameter for dispersion engineering, and we will particularly focus on resonators that have a large radius to width ratio and that are not well described using whispering-gallery-modes $[\underline{17}, \underline{18}]$. The analytics can help provide additional insight over numerical simulations and should be seen as a complement to more precise numerical methods. We aim to present a guide for determining all mode-dependent linear parameters, c.f. [19-23], and nonlinear parameters that are of interest for designing microresonators for nonlinear FWM applications, such as frequency comb generation. This includes dispersion, nonlinear and coupling coefficients, as well as resonance frequencies, free-spectral range (FSR) 
and $Q$-factor. The dynamical evolution of the intracavity field can then be described using either the formalism of the driven and damped nonlinear Schrödinger equation $[\underline{16}, 24,25]$ (Lugiato-Lefever equation), or the coupled mode model $[26,27]$.

We begin the next section by deriving an analytical solution for the eigenmode/eigenvalue problem of a planar ring resonator in a cylindrical geometry. We consider examples of silicon resonators with the material refractive index determined by a Sellmeier formula. We also derive an approximate rectangular solution of the eigenmode/eigenvalue problem for the important case when the width of the resonator is small in comparison to the radius, and develop an explicit analytical approximation for this case. Finally, we demonstrate how the FSR and dispersion properties can be determined using knowledge of the resonance frequencies. Next, in Section $\underline{3}$, we consider the influence of the mode profile on the effective nonlinear Kerr coefficient, and present an expression for estimating the mode area. Waveguide coupling is treated in Section $\underline{4}$. We use a quasi-TE/TM approximation to derive coupling coefficients, with examples given for ring and racetrack geometries. We additionally consider estimates for the resonator $Q$-factor. The final section contains conclusions and a discussion of the applicability of the analytical model.

\section{EIGENMODE ANALYSIS}

In this section, we begin by deriving an analytical solution for the eigenmode/eigenvalue problem of a ring resonator with an axisymmetric cylindrical geometry, assuming that the in-plane and perpendicular components of the fields are separable [28]. This allows for implicit analytical expressions for the electric and magnetic fields of the modes, as well as their resonance frequencies, to be found. An accurate knowledge of the resonance frequencies can be used to determine the complete dispersion profile, including both material and geometric contributions.

Using the assumption of separable fields, we employ the effective index method and introduce an auxiliary refractive index $n_{e}$ to separate the in-plane and perpendicular field dependence. The auxiliary refractive index is a quantity, which lies in between the material refractive index $n_{1}$ and the effective index $n_{\text {eff }}$ of the core, and that is useful on its own for numerical simulations (e.g., Finite Element Method or Finite Difference Time Domain) of resonator and waveguide structures, since it can be used to reduce the dimensionality of the problem from three to two dimensions.

There are two resonator mode families with the electric field polarized predominantly either in the radial or perpendicular direction. Using the conventional notation, these will be referred to as $E_{p q}^{x}$ and $E_{p q}^{y}$ [29], where the subscripts $p$ and $q$ denote the number of maxima in the in-plane $x$ (or radial $r$ ) and perpendicular $y$ direction, respectively, as shown in Fig. 1. We will present a detailed analysis for the $E_{p q}^{x}$ mode and present only the result for the $E_{p q}^{y}$ mode, which is derived analogously. Our starting point is the wave equation for the magnetic field in a cylindrical geometry, i.e.,

$$
\left(\nabla^{2}-\frac{n^{2}}{c^{2}} \frac{\partial^{2}}{\partial t^{2}}\right) \mathbb{H}=0,
$$

which can be derived from Maxwell's equations in the absence of free charges and currents. Following Marcatili's

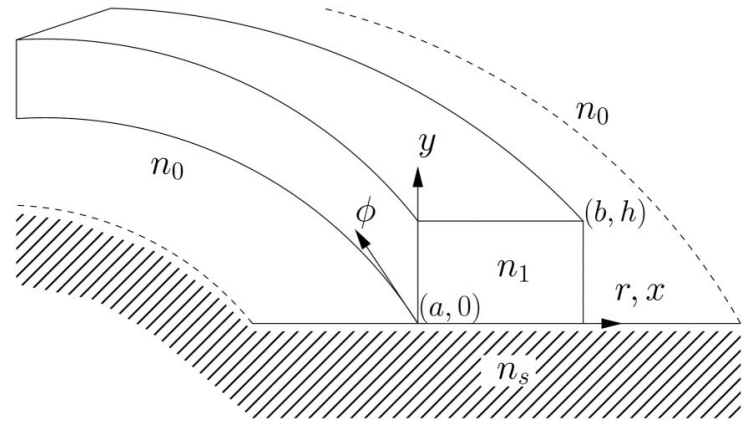

Fig. 1. Cross section showing layer structure of the ring waveguide.

approach [29] we set $H_{r}=0$ and consider the equation for the $H_{y}$ field component. Assuming a plane wave solution $H_{y}(r, y, \phi)=H_{y}(r, y) e^{i(\omega t-m \phi)}$ for the angular dependence, the wave equation can then be written as

$$
\left[\frac{\partial^{2}}{\partial r^{2}}+\frac{1}{r} \frac{\partial}{\partial r}+\frac{\partial^{2}}{\partial y^{2}}+\left(k_{0}^{2} n^{2}-\frac{m^{2}}{r^{2}}\right)\right] H_{y}(r, y)=0
$$

where the angular periodicity requires that the mode number $m$ is a constant, which is related to the propagation constant $\beta$ as $m=\beta r_{0}$ with $r_{0}$ being the (mean) radius of the resonator.

\section{A. Radial Mode Solution}

We now make the critical assumption that the field is separable, i.e., $H_{y}(r, y)=R(r) Y(y)$, and employ the effective index method to obtain separate equations for the in-plane factor $R(r)$ and perpendicular factor $Y(y)$ :

$$
\begin{gathered}
\frac{1}{r} \frac{d}{d r}\left(r \frac{d R}{d r}\right)+\left(k_{0}^{2} n_{e}^{2}-\frac{m^{2}}{r^{2}}\right) R=0 \\
\frac{d^{2} Y}{d y^{2}}+k_{0}^{2}\left(n^{2}-n_{e}^{2}\right) Y=0
\end{gathered}
$$

where we have introduced the auxiliary refractive index $n_{e}$, which is obtained from the separation constant $k_{0}^{2} n_{e}^{2}$. Equations (3) and (4) are to be solved self-consistently to obtain the vacuum wavenumber $k_{0}(m)=2 \pi / \lambda_{0}$ and the auxiliary refractive index $n_{e}=n_{e}(m)$ for each resonant mode with mode number $m$. Note that the auxiliary index is sometimes also referred to as an effective refractive index, but we will use the term auxiliary index to avoid confusion with the effective index $n_{\text {eff }}=n_{\text {eff }}(m)$ that is related to the propagation constant $\beta(m)=k_{0} n_{\text {eff }}$.

We consider a ring waveguide that has a three-layer sandwich structure in the perpendicular direction, consisting of: a substrate, a core layer, and a cladding, as illustrated by the cross section in Fig. 1 . The general solution of the radial Eq. (3) for each region, which satisfies the physical boundary conditions of being finite on-axis and vanishing at infinity, is given by

$$
\begin{gathered}
R_{0}(r)=A J_{m}\left(k_{r} r\right)+B Y_{m}\left(k_{r} r\right), \quad a \leq r \leq b, \\
R_{-}(r)=C I_{m}\left(\alpha_{r} r\right), \quad 0 \leq r \leq a, \\
R_{+}(r)=D K_{m}\left(\alpha_{r} r\right), \quad r \geq b,
\end{gathered}
$$


where $J_{m}\left(k_{r} r\right)$ and $Y_{m}\left(k_{r} r\right)$ are Bessel functions of the first and second kind while $I_{m}\left(\alpha_{r} r\right)$ and $K_{m}\left(\alpha_{r} r\right)$ are modified Bessel functions of the first and second kind, respectively. The coefficients $A, B, C$, and $D$ are constants that will be determined by the continuity requirement of the $H_{y}$ component of the magnetic field and of the tangential components $E_{\phi}$ and $H_{\phi}$, which, using the assumption of $H_{r}=0$, are obtained from Maxwell's equations in cylindrical coordinates as

$$
E_{\phi}(r, y)=\frac{i}{\omega \varepsilon_{0} n^{2}} \frac{\partial H_{y}(r, y)}{\partial r}, \quad H_{\phi}(r, y)=-\frac{i r}{\beta} \frac{\partial H_{y}(r, y)}{\partial y}
$$

for the dominant $H_{y}$ component of the $E_{p q}^{x}$ modes while the $H_{r}$ component of the $E_{p q}^{y}$ modes satisfies

$E_{\phi}(r, y)=-\frac{i}{\omega \varepsilon_{0} n^{2}} \frac{\partial H_{r}(r, y)}{\partial y}, \quad H_{\phi}(r, y)=-\frac{i}{\beta} \frac{\partial\left(r H_{r}(r, y)\right)}{\partial r}$

It should be noted that the modes of a microring resonator are somewhat different from the whispering-gallery-modes (WGMs) of a uniformly filled cylindrical microdisk resonator. The presence of an inner boundary wall implies that the resonator has a different topology (toroidal), which forces the field of the microring to be exponentially decaying also in the central cladding region. While the cylindrical WGM approximation can still be appropriate in certain limits, it is generally unsuitable for resonators possessing a large radius to width ratio. This can clearly be seen from the resonance mode profiles in the example of Fig. $\underline{6}$, which shows both numerically and analytically calculated modes at a wavelength of $2.5 \mu \mathrm{m}$ for a $1 \mu \mathrm{m}$ wide silicon microring resonator with a mean radius of $50 \mu \mathrm{m}$. Figure 6 shows the cylindrical WGMs to be a poor approximation in this limit, with the mode extending far into the inner cladding region. The WGMs have peak amplitudes close to the inner boundary wall and exhibit large shifts in mode numbers compared with the numerical mode solver solutions that are centered in the waveguide. The approximation for the mode area is also poor since the WGMs are much wider than the actual width of the waveguide. Note however, that although $R(r)$ is an exact solution of the radial Eq. (3), it is only an approximation of the actual magnetic field. The remaining electromagnetic field components will generally not satisfy the vector wave equation (1) exactly.

The coefficients of the eigenmodes can be obtained as

$$
B=-A \frac{J_{m}^{\prime}\left(k_{r} a\right)-\eta\left(I_{m}^{\prime}\left(\alpha_{r} a\right) / I_{m}\left(\alpha_{r} a\right)\right) J_{m}\left(k_{r} a\right)}{Y_{m}^{\prime}\left(k_{r} a\right)-\eta\left(I_{m}^{\prime}\left(\alpha_{r} a\right) / I_{m}\left(\alpha_{r} a\right)\right) Y_{m}\left(k_{r} a\right)},
$$

and

$$
C=\frac{A J_{m}\left(k_{r} a\right)+B Y_{m}\left(k_{r} a\right)}{I_{m}\left(\alpha_{r} a\right)}, \quad D=\frac{A J_{m}\left(k_{r} b\right)+B Y_{m}\left(k_{r} b\right)}{K_{m}\left(\alpha_{r} b\right)},
$$

where the amplitude $A$ is arbitrary, and the simultaneous solution of the continuity requirements at the medium boundaries $a$ and $b$ gives a consistency condition for the mode:

$$
\begin{aligned}
& {\left[J_{m}^{\prime}\left(k_{r} a\right)-\eta \frac{I_{m}^{\prime}\left(\alpha_{r} a\right)}{I_{m}\left(\alpha_{r} a\right)} J_{m}\left(k_{r} a\right)\right]\left[Y_{m}^{\prime}\left(k_{r} b\right)-\eta \frac{K_{m}^{\prime}\left(\alpha_{r} b\right)}{K_{m}\left(\alpha_{r} b\right)} Y_{m}\left(k_{r} b\right)\right]} \\
& -\left[Y_{m}^{\prime}\left(k_{r} a\right)-\eta \frac{I_{m}^{\prime}\left(\alpha_{r} a\right)}{I_{m}\left(\alpha_{r} a\right)} Y_{m}\left(k_{r} a\right)\right] \\
& \quad \times\left[J_{m}^{\prime}\left(k_{r} b\right)-\eta \frac{K_{m}^{\prime}\left(\alpha_{r} b\right)}{K_{\beta}\left(\alpha_{r} b\right)} J_{m}\left(k_{r} b\right)\right]=0
\end{aligned}
$$

where $k_{r}=k_{0} n_{e}, \alpha_{r}=k_{0} n_{0}$ and $\eta=n_{1} / n_{0}$ for the $E_{p q}^{x}$ mode while $\eta=n_{0} / n_{1}$ for the $E_{p q}^{y}$ mode. The cylindrical WGMs can be obtained from the radial mode solution in the limit of $a \rightarrow 0$ with $B=C=0$ and requires that the solution satisfies the consistency condition $J_{m}^{\prime}\left(k_{r} b\right) K_{\beta}\left(\alpha_{r} b\right)=$ $\eta K_{m}^{\prime}\left(\alpha_{r} b\right) J_{m}\left(k_{r} b\right)=0$.

The general solution for each region of Eq. (ㅁ) is similarly given by

$$
\begin{gathered}
Y_{+}(y)=\cos \left(k_{y} h-\psi\right) e^{-\alpha_{0}(y-h)}, \quad y \geq h, \\
Y_{0}(y)=\cos \left(k_{y} y-\psi\right), \quad 0 \leq y \leq h, \\
Y_{-}(y)=\cos (\psi) e^{\alpha_{s} y}, \quad y \leq 0,
\end{gathered}
$$

where $\quad k_{y}=k_{0} \sqrt{n_{1}^{2}-n_{e}^{2}}, \quad \alpha_{s}=k_{0} \sqrt{n_{e}^{2}-n_{s}^{2}} \quad$ and $\quad \alpha_{0}=$ $k_{0} \sqrt{n_{e}^{2}-n_{0}^{2}}$ [30]. Requiring the fields $H_{y}$ (or $H_{r}$ ) to be continuous at the boundaries results, in accordance with Eq. (8) [or Eq. (9) for the $E_{p q}^{y}$ mode], in another consistency requirement:

$$
k_{y} h=(q-1) \pi+\arctan \left(\xi_{1} \frac{\alpha_{s}}{k_{y}}\right)+\arctan \left(\xi_{2} \frac{\alpha_{0}}{k_{y}}\right),
$$

where $\xi_{1}=\xi_{2}=1$ for the $E_{p q}^{x}$ mode while $\xi_{1}=n_{1}^{2} / n_{s}^{2}$ and $\xi_{2}=n_{1}^{2} / n_{0}^{2}$ for the $E_{p q}^{y}$ mode and we have used that $\tan \psi=\alpha_{s} / k_{z}$.

The simultaneous solution of Eqs. (12) and (16) allows the unique wavenumber $k_{0}(m)$ and auxiliary refractive index $n_{e}(m)$ of a given resonance to be determined, with each resonance corresponding to a particular mode with mode number $m$. Once the resonance frequency of the mode $m$ is known, the effective refractive index can be determined from the relation $n_{\text {eff }}=m /\left(k_{0} r_{0}\right)$; i.e., the requirement that the circumference of the resonator corresponds to an integer number of wavelengths. Note also that Eq. (16) can be used to obtain an explicit relation for $k_{0}$ as a function of $n_{e}$, under the assumption that $n_{1}$ is frequency-independent.

\section{B. Frequency-Dependent Refractive Index}

To accurately determine the resonance frequencies and the dispersion profile, it is necessary to self-consistently take into account the frequency dependence of the refractive index of the core material. This can most easily be done using a Sellmeier equation that expresses the core index as function of the vacuum wavenumber, i.e., $n_{1}=n_{1}\left(k_{0}\right)$. The wavenumber dependent refractive index of silicon can, e.g., be modeled using the Sellmeier formula

$$
n_{1}\left(k_{0}\right)=\sqrt{B_{0}+B_{1} k_{0}^{2}+B_{2} k_{0}^{4}},
$$


where $B_{0}=11.713, \quad B_{1}=1.92826 \cdot 10^{-14} \mathrm{~m}^{2}, \quad$ and $\quad B_{2}=$ $1.89593 \cdot 10^{-28} \mathrm{~m}^{4}$. This formula represents a least square fit of 19 measurement values between 1.12 and $5 \mu \mathrm{m}$, taken from Ref. [31].

\section{Rectangular Approximation}

The previous solution for the eigenmodes and eigenfrequencies is applicable to cylindrically symmetric ring resonators with arbitrary radii, and must be used when there is an appreciable radial shift of the peak intensity position with respect to the center of the waveguide. However, if the radius is sufficiently large in comparison with the width of the resonator, it becomes possible to approximate the cross section of the ring waveguide with the rectangular cross section of a straight waveguide. This simplifies the analysis considerably, since the radial mode solution can now be expressed using trigonometric functions, instead of the more cumbersome Bessel functions. This approximation also makes it possible to treat resonators with a more complicated geometry than ring resonators within the same formalism, i.e., resonators lacking an axial symmetry such as, e.g., racetracks or folded resonators, provided that the local radius of curvature of any bends remains sufficiently large.

The reasoning behind this approximation can be made more precise by noting that the radial factor should satisfy the following approximate equation at the center of the ring where the field has its greatest magnitude:

$$
\begin{gathered}
{\left[\frac{d^{2} R}{d r^{2}}+\frac{1}{r} \frac{d R}{d r}+\left(k_{0}^{2} n_{e}^{2}-\frac{m^{2}}{r^{2}}\right) R\right]_{r=r_{0}}} \\
\approx-k_{x}^{2}+i \frac{k_{x}}{r_{0}}+\left(k_{0}^{2} n_{e}^{2}-\frac{m^{2}}{r_{0}^{2}}\right) \approx 0 .
\end{gathered}
$$

Here, we have used that the solution for the rectangular case is given by $R(r) \propto \exp \left(i k_{x} r\right)$. It is easily seen that the second term on the left-hand side can be neglected with respect to the first term if the condition $\left|k_{x} r_{0}\right| \gg 1$ is satisfied, which shows that Eq. (18) can be approximated as

$$
\frac{d^{2} X}{d x^{2}}+\left(k_{0}^{2} n_{e}^{2}-\beta^{2}\right) X=0
$$

where we have replaced the radial function $R(r)$ with the inplane function $X(x)$ and used that $m=\beta r_{0}$. In the following we will, therefore, assume that the magnetic field factors as $H_{y}(x, y)=X(x) Y(y)$, and replace Eq. (3) with Eq. (19) while Eq. (4) for the perpendicular factor remains the same.

The general solution of Eq. (19) is similar to that of Eqs. (13-15) and it is found to be given by

$$
\begin{gathered}
X_{-}=A \cos \left(k_{x} a-\phi\right) e^{\alpha_{x}(x-a)}, \quad 0 \leq x \leq a, \\
X_{0}=A \cos \left(k_{x} x-\phi\right), \quad a \leq x \leq b, \\
X_{+}=A \cos \left(k_{x} b-\phi\right) e^{-\alpha_{x}(x-b)}, \quad x \geq b .
\end{gathered}
$$

The boundary conditions for the $E_{p q}^{x}\left(E_{p q}^{y}\right)$ mode require that $H_{y}\left(H_{x}\right)$, as well as the transverse components $E_{\phi} \propto\left(1 / n^{2}\right) \partial H_{y} / \partial x \quad\left(H_{\phi} \propto \partial H_{x} / \partial x\right)$ and $H_{\phi} \propto \partial H_{y} / \partial y$ $\left(E_{\phi} \propto\left(1 / n^{2}\right) \partial H_{x} / \partial y\right)$, should be continuous at the interfaces.
This leads to an equation for the phase $\phi=k_{x}(a+b) / 2+$ $l \pi / 2$ and the following consistency condition:

$$
k_{x}(b-a)=(p-1) \pi+2 \arctan \left(\eta \frac{\alpha_{x}}{k_{x}}\right),
$$

where we now have $k_{x}=\sqrt{k_{0}^{2} n_{e}^{2}-\beta^{2}}$ and $\alpha_{x}=\sqrt{\beta^{2}-k_{0}^{2} n_{0}^{2}}$. The coefficient $\eta=n_{1}^{2} / n_{0}^{2}$ for the $E_{p q}^{x}$ mode while $\eta=1$ for the $E_{p q}^{y}$ mode. The propagation constant $\beta$ can be determined from the condition that the circumference $L$ of the resonator should correspond to an integer number $m$ of wavelengths, i.e., $\beta L=2 \pi m$. This condition simplifies to $\beta r_{0}=m$ for a ring resonator, but is also valid for resonators with an arbitrary folded geometry. The effective refractive index, corresponding to a given resonance frequency of a particular mode, is obtained from the propagation constant of that mode as

$$
n_{\mathrm{eff}}=\beta / k_{0}=2 \pi m /\left(k_{0} L\right) .
$$

\section{Analytical Approximation}

Using the rectangular approximation, it is also possible to obtain some explicit analytical approximations for the effective and auxiliary refractive indices as a function of the vacuum wavenumber. In particular, we can use the approximation $\arctan (x) \approx(3 x / 2) / \sqrt{3+x^{2}}$ to find solutions of the consistency conditions of Eqs. (16) and (23). We find that the auxiliary and effective refractive indices of the $E_{11}^{x}$ mode can be written as

$$
\begin{gathered}
n_{e}^{2}\left(k_{0}\right)=n_{1}^{2}\left(k_{0}\right)+F\left[n_{1}\left(k_{0}\right)^{2}-\frac{n_{0}^{2}+n_{s}^{2}}{2}, 1, k_{0} h\right], \\
n_{\mathrm{eff}}^{2}\left(k_{0}\right)=n_{e}^{2}\left(k_{0}\right)+F\left[n_{e}^{2}\left(k_{0}\right)-n_{0}^{2}, \frac{n_{1}^{2}\left(k_{0}\right)}{n_{0}^{2}}, k_{0}(b-a)\right],
\end{gathered}
$$

and the refractive indices of the $E_{11}^{y}$ mode can be written as

$$
\begin{gathered}
n_{e}^{2}\left(k_{0}\right)=n_{1}^{2}\left(k_{0}\right)+F\left[n_{1}^{2}\left(k_{0}\right)-\frac{n_{0}^{4}+n_{s}^{4}}{n_{0}^{2}+n_{s}^{2}}, \frac{2 n_{1}^{2}\left(k_{0}\right)}{n_{0}^{2}+n_{s}^{2}}, k_{0} h\right], \\
n_{\text {eff }}^{2}\left(k_{0}\right)=n_{e}^{2}\left(k_{0}\right)+F\left[n_{e}^{2}\left(k_{0}\right)-n_{0}^{2}, 1, k_{0}(b-a)\right],
\end{gathered}
$$

with the function $F$ defined by

$F[a, b, c]=\frac{9 b^{2}}{2 c^{2}\left(3-b^{2}\right)}\left(1+\frac{c^{2} a}{9}-\sqrt{\left(1+\frac{c^{2} a}{9}\right)^{2}+\frac{4\left(3-b^{2}\right) c^{2} a}{9 b^{2}}}\right)$.

These approximations agree very well with the effective refractive index that has been numerically calculated for a rectangular silicon waveguide (Figs. $\underline{2}$ and $\underline{3}$ ). Interestingly, the explicit analytical approximation of the $E_{11}^{y}$ is in better agreement with the numerical mode solver solution than the numerical solution of the consistency requirement that it approximates. Note also that the refractive index of the $E_{11}^{y}$ mode approaches the substrate index $n_{s}=1.54$ for wavelengths close to $4 \mu \mathrm{m}$. 
However, one should be careful about using the approximations for the effective refractive index to estimate cavity dispersion and zero-dispersion wavelengths. The reason for this is that the dispersion depends on the second derivative, which is more sensitive than the function value of the propagation constant. One should, therefore, verify the analytical approximations with the numerical mode solver solution (Figs. $\underline{4}$ and $\underline{5}$ ).

\section{E. Dispersion}

Inserting Eq. (24) into Eq. (23) allows the vacuum wavenumber $k_{0}=k_{0}(m)$ and the auxiliary refractive index $n_{e}=n_{e}(m)$ of the mode to be determined as the simultaneous solution of Eqs. (16) and (23). The resonance frequency can then be obtained as $\omega_{m}=k_{0} c$, together with the effective refractive index for that frequency from Eq. (24).

Alternatively, it is also possible to solve Eqs. (16) and (23) for $\beta=\beta\left(k_{0}\right)$ and $n_{e}=n_{e}\left(k_{0}\right)$ as continuous functions of the wavenumber. This is similar to how the propagation constant

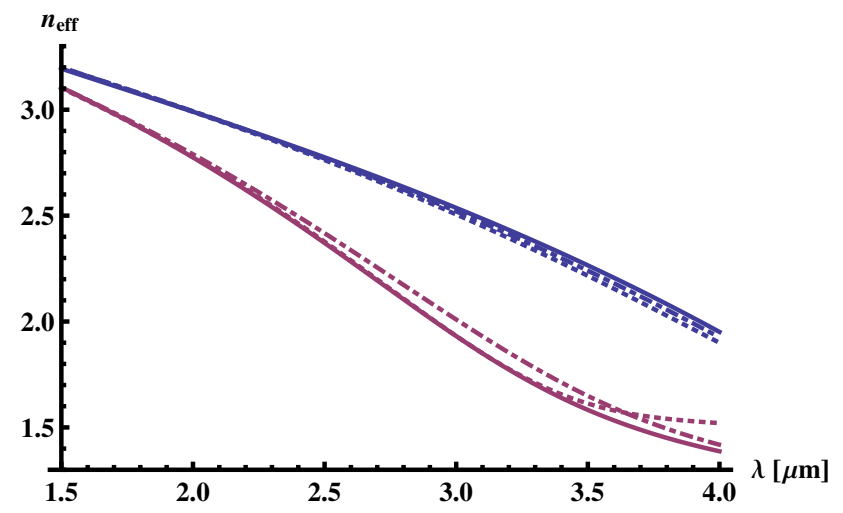

Fig. 2. Effective refractive index as a function of wavelength for a silicon ring resonator with $a=49.5 \mu \mathrm{m}, b=50.5 \mu \mathrm{m}$, and $h=0.5 \mu \mathrm{m}$. Fully drawn lines are explicit analytical approximations, while dotted lines are numerical solutions of the consistency requirements; dashed-dotted lines are numerical mode solver solutions. The upper solutions (blue color) signify the $E_{11}^{x}$ mode and the lower solution (red color) the $E_{11}^{y}$ mode.

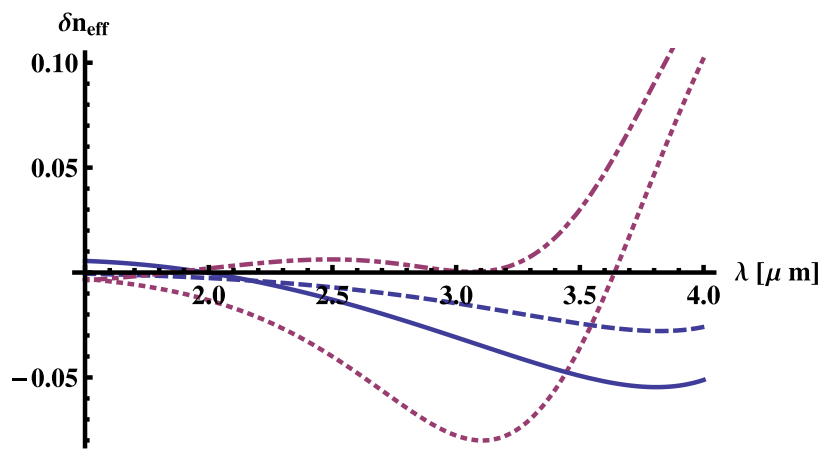

Fig. 3. Differences between the analytical approximations and mode solver solution for the effective refractive index plotted in Fig. 2. The fully drawn (blue) line signifies the difference of the explicit analytical approximation of the $E_{11}^{x}$ mode, while the dashed (blue) line is the difference of the numerical solution of the consistency requirement for the $E_{11}^{x}$ mode. The dashed-dotted (red) line is the corresponding difference of the explicit analytical approximation of the $E_{11}^{y}$ mode while the dotted (red) line is the difference of the numerical solution of the consistency requirement for the $E_{11}^{y}$ mode. is usually determined for straight waveguides. The eigenfrequencies are then found as those frequencies that satisfy $\beta L=2 \pi m$. By differentiating $\beta$ with respect to frequency, i.e.,

$$
\beta_{n}=\frac{d^{n} \beta}{d \omega^{n}}{ }_{\omega=\omega_{m 0}}
$$

one can obtain the FSR $\omega_{\mathrm{FSR}}=1 /\left(\beta_{1} r_{0}\right)$, as well as dispersion coefficients $\beta_{2}, \beta_{3}$, etc.

Once the set of resonance frequencies $\omega_{m}$ is known, they can be used to determine the FSR and dispersion profile of the resonator. For this purpose, it is helpful to introduce a finite difference operator $\Delta \omega_{m}=\omega_{m+1 / 2}-\omega_{m-1 / 2}$, with half-integer mode numbers, defined as $\omega_{m+1 / 2}=\left(\omega_{m+1}+\omega_{m}\right) / 2$, which can be used to find the coefficients $D_{k}=\Delta^{k} \omega_{m}$ of the following Taylor expansion for the resonance frequencies around the pump frequency $\omega_{m 0}$, viz.

$$
\begin{aligned}
\omega_{m}= & \omega_{m 0}+D_{1}\left(m-m_{0}\right)+\frac{1}{2} D_{2}\left(m-m_{0}\right)^{2} \\
& +\frac{1}{6} D_{3}\left(m-m_{0}\right)^{3}+\ldots
\end{aligned}
$$

Here, the coefficient $D_{1}$ is the FSR while $D_{2}$ is related to the second-order dispersion coefficient, with $\beta_{2}=-D_{2}\left(\tau_{0} / D_{1}^{2}\right)\left(\tau_{0}\right.$ being the round-trip time) in the formalism of the LugiatoLefever equation [24]. Calculating the second-order difference $D_{2}=\Delta^{2} \omega_{m}$ for different $m$ allows the complete dispersion

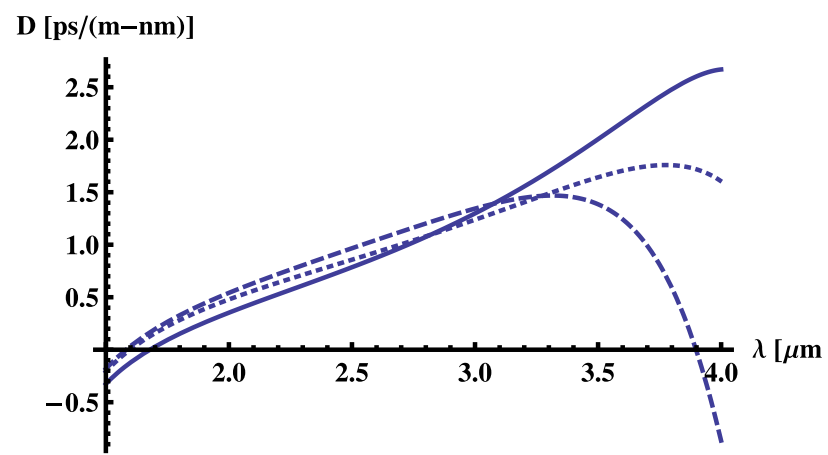

Fig. 4. Dispersion $D$ for the $E_{11}^{x}$ mode of a silicon ring resonator with $a=49.5 \mu \mathrm{m}, b=50.5 \mu \mathrm{m}$, and $h=0.5 \mu \mathrm{m}$. Fully drawn lines are explicit analytical approximations, while dotted lines are numerical solutions of the consistency requirements and dashed lines are numerical mode solver solutions.

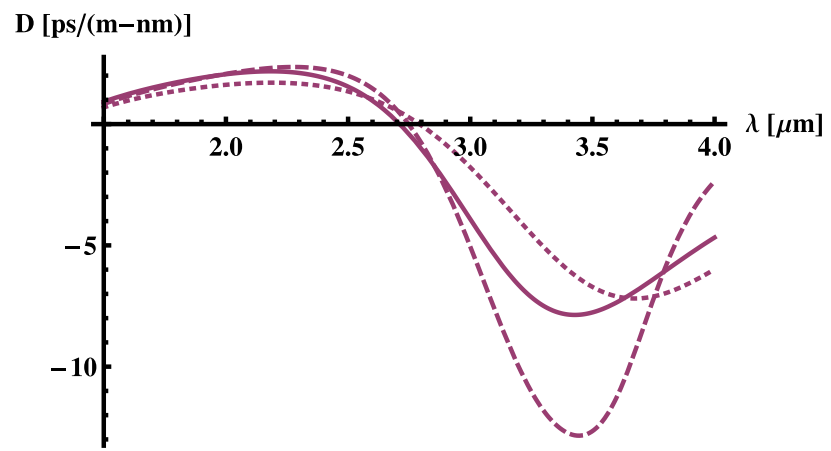

Fig. 5. Dispersion $D$ for the $E_{11}^{y}$ mode of a silicon ring resonator with the same parameters as in Fig. 4 . 
profile to be found. By changing the device geometry and, in particular, the width and height of the ring waveguide, it is thus possible to engineer the dispersion profile and optimize the resonator design for nonlinear applications. Wavelength ranges and magnitudes of normal or anomalous dispersion can be determined from $D_{2}$, as well as any zero-dispersion wavelengths. We emphasize that not only the repetitionrate/FSR, but also the dispersion profile is of critical importance for frequency comb generation and nonlinear FWM applications, since it allows tuning of the modulational instability process. An optimal design criterion can, e.g., be to have anomalous cavity dispersion, i.e., $D_{2}>0$, with a flat profile and a magnitude that makes the peak modulational instability gain overlap with a chosen sideband pair. While an in-depth study of the dependence of the dispersion properties on the device geometry is of great practical interest, it is, however, beyond the scope of the current article. More detailed investigations and comparisons of the resonance spectrum with other types of microcavities [18,32], will instead be the topic of future work.

In Figs. $\underline{4}$ and $\underline{5}$, we show a comparison of analytically and numerically calculated profiles of the second-order dispersion parameter $D=-(\lambda / c) d^{2} n_{\text {eff }} / d \lambda^{2}$ for a silicon ring resonator. The resonator is assumed to have a radius of $50 \mu \mathrm{m}$, a width of $1 \mu \mathrm{m}$, and a height of $0.5 \mu \mathrm{m}$. The material refractive index of the core layer is assumed to be given by Eq. (17) while the cladding (air) and substrate $\left(\mathrm{SiO}_{2}\right)$ have constant refractive indices of $n_{0}=1$ and $n_{s}=1.54$, respectively. The numerical dispersion profile was obtained by applying a commercial finite element mode solver (COMSOL) to a straight waveguide, and the accuracy was ascertained by successively refining the mesh until the eigenvalues converged. However, one should instead use a radial mode solver [33] if the inequality $\left|k_{x} r_{0}\right| \gg 1$ is not satisfied.

\section{NONLINEAR PROPERTIES}

The generation of optical frequency combs relies on modulational instability and the nonlinear FWM process to generate new frequency components from the CW pump mode. The efficiency of this process depends on the magnitude of the nonlinear coefficient. The Kerr coefficient of a microring resonator in the formalism of the driven and damped NLS equation is inversely proportional to the effective mode area $A_{m}$ of the resonator, i.e.,

$$
\gamma=\frac{\omega_{m 0} n_{2}}{c A_{m 0}} L
$$

with $n_{2}$ being the nonlinear refractive index coefficient $\left(n \approx n_{0}+n_{2} I\right.$ ). The mode area can be related to the mode volume as $V_{m 0}=A_{m 0} L$. It is critical to have a small mode area to get a large field enhancement effect and efficient frequency conversion.

In this section, we make a simple approximation of the effective mode area of a microring resonator, using the rectangular mode approximation. Since we are interested in determining the magnitude of the nonlinear Kerr coefficient, we will use the definition:

$$
A_{m}=\frac{\left(\int\left|H_{x, y}(x, y)\right|^{2} \mathrm{~d} A\right)^{2}}{\int\left|H_{x, y}(x, y)\right|^{4} \mathrm{~d} A} .
$$

The rectangular approximation is motivated when the waveguide is narrow and the ring radius is large. Because of the high degree of localization of the mode inside of the core region due to the high index contrast, as well as the absence of nonlinear effects in the air cladding, we may neglect the field outside of the core area. We then find that the effective mode area can be approximated from Eq. (33) as

$$
A_{m} \approx \frac{4}{9}\left((b-a)+\frac{2 \eta \alpha_{x}}{k_{x}^{2}+\eta^{2} \alpha_{x}^{2}}\right)\left(h+\frac{\xi_{1} \alpha_{s}}{k_{z}^{2}+\xi_{1}^{2} \alpha_{s}^{2}}+\frac{\xi_{2} \alpha_{0}}{k_{z}^{2}+\xi_{2}^{2} \alpha_{0}^{2}}\right),
$$

which is valid both for the $E_{p q}^{x}$ mode and the $E_{p q}^{y}$ modes, using the respective definitions of $\eta$ and $\xi_{i}$. The above expression tends to overestimate the core contribution somewhat, but agrees reasonably well with the more precise values that can be found numerically by integrating the mode profile from the mode solver solution (Figs. $\underline{6}$ and $\underline{7}$ ).

\section{WAVEGUIDE COUPLING}

Besides considering the FSR and dispersion properties, it is also necessary to take into account the coupling of light into and out of the resonator. In this paper, we assume that the coupling occurs due to an evanescent field overlap between the resonator and either one or two slab waveguides that are made of the same material as the resonator. This allows coupling coefficients, as well as $Q$-value and coupling regimes, to be estimated. We further assume that the radius-to-width ratio is sufficiently large that the radial dependence can be neglected.

The effective index method allows the in-plane field dependence to be separated from the perpendicular field dependence. This can be used to reduce the dimensionality of the problem from three to two dimensions if the auxiliary refractive index is used instead of the material refractive

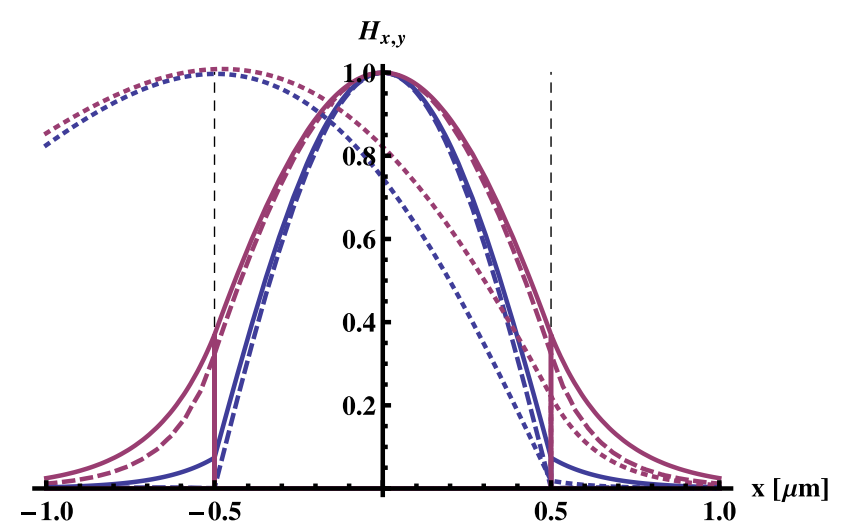

Fig. 6. Comparison of cylindrical whispering-gallery-modes, explicit analytical solutions using the rectangular approximation, and numerical mode solver solutions for the normalized mode profiles of resonant modes at $\lambda=2.5 \mu \mathrm{m}$ in the $x$-direction of a silicon ring resonator. Dotted lines are WGMs, fully drawn lines are explicit analytical approximations, and dashed lines are numerical mode solver solutions. The lower solution (blue color) signifies the $H_{y}$ component of the $E_{11}^{x}$ mode and the upper solution (red color) the $H_{x}$ component of the $E_{11}^{y}$ mode. The $x$-scale is relative to the ring radius $r_{0}$. 


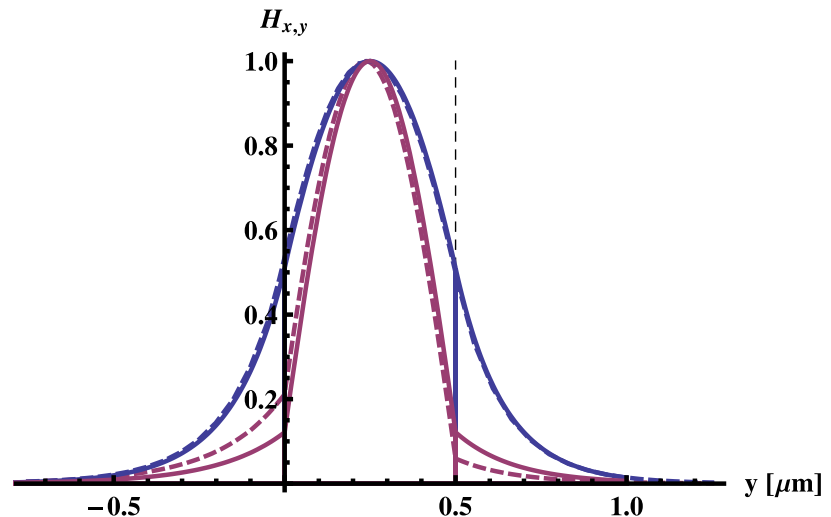

Fig. 7. Comparison of explicit analytical and mode solver solutions for the normalized mode profiles in the $y$-direction for resonant modes at $\lambda=2.5 \mu \mathrm{m}$ of the same silicon ring resonator as in Fig. (6). The upper solution (blue color) signifies the $H_{y}$ component of the $E_{11}^{x}$ mode and the lower solution (red color) the $H_{x}$ component of the $E_{11}^{y}$ mode.

index for the two dimensional case. The $E_{p q}^{x}$ mode can then be approximated as a TM-mode while the $E_{p q}^{y}$ mode can be approximated as a TE-mode. Using the auxiliary refractive index, one finds that the in-plane TE-mode has the same dispersion relation as the $E_{p q}^{y}$ mode, i.e., Eq. (19) with $\eta=1$. The effective index and the resonance frequencies should, therefore, be the same as those that can be found using the rectangular approximation. For the TM-mode, which corresponds to the $E_{p q}^{x}$ mode, one finds that the TM-mode satisfies a dispersion relation similar to Eq. (19), but with $\eta=n_{e}^{2} / n_{0}^{2}$. The argument of the arctan function will, therefore, differ from that of the $E_{p q}^{x}$ mode, which has $\eta=n_{1}^{2} / n_{0}^{2}$. However, this is still a reasonable approximation as long as the argument is large.

The local coupling coefficient between the bus and ring waveguide at a particular point in a slab geometry can, according to coupled mode theory [34], be calculated from the overlap integral

$$
\hat{\kappa}[s(\phi)]=\frac{\omega \varepsilon_{0}}{4 I} \int_{-\infty}^{\infty}\left(n_{e}^{2}-n_{0}^{2}\right) \mathbb{E}_{1}^{*} \cdot \mathbb{E}_{2} \mathrm{~d} x,
$$

where

$$
I=\frac{1}{4} \int_{-\infty}^{\infty}\left(\mathbb{E}_{1}^{*} \times \mathbb{H}_{1}+\mathbb{E}_{1} \times \mathbb{H}_{1}^{*}\right) \cdot \hat{\phi} \mathrm{d} x
$$

is the mode power.

The lumped coupling coefficient $\kappa$ is subsequently found by integrating the local coupling coefficient along the length of the waveguide while taking into account the varying separation distances $s=s(\phi)$, and is given by the expression

$$
\kappa=\int_{-\infty}^{\infty} \hat{\kappa}[s(\phi)] e^{-i \Delta \beta \phi} \mathrm{d} \phi,
$$

where $\Delta \beta=\beta_{1}-\beta_{2}$ corresponds to the mismatch between the propagation constants of the two waveguides.

Assuming that the two waveguides are identical we find the local coupling coefficient to be given by

$$
\hat{\kappa}_{y}=\frac{2 k_{x}^{2} \alpha_{x}}{\beta\left(k_{x}^{2}+\alpha_{x}^{2}\right)\left(\frac{b-a}{2}+\frac{2}{\alpha_{x}}\right)} \exp \left[-\alpha_{x}(2 s-(b-a))\right],
$$

for the TE-mode $\left(E_{p q}^{y}\right)$ and

$$
\begin{aligned}
\hat{\kappa}_{x}= & \frac{\left(n_{e} / n_{0}\right)^{2} 2 k_{x}^{2} \alpha_{x}}{\beta\left(k_{x}^{2}+\left(n_{e} / n_{0}\right)^{4} \alpha_{x}^{2}\right)\left(\frac{b-a}{2}+\frac{2}{\alpha_{x}} \frac{n_{e}^{2}}{n_{0}^{2}} \frac{k_{x}^{2}+\alpha_{x}^{2}}{k_{x}^{2}+\left(n_{e} / n_{0}\right)^{4} \alpha_{x}^{2}}\right)} \\
& \times \exp \left[-\alpha_{x}(2 s-(b-a))\right],
\end{aligned}
$$

for the TM-mode $\left(E_{p q}^{x}\right)$, c.f. [35].

The lumped coupling coefficient depends on how the separation distance varies with position. If we consider e.g., a ring or racetrack shaped resonator coupled to a straight waveguide, we find that the separation distance can be approximated by a function

$$
\begin{array}{ll}
s(\phi)=s_{0}, & |\phi| \leq l_{0} \\
s(\phi)=s_{0}+\left(|\phi|-l_{0}\right)^{2} /\left(2 r_{0}\right), & |\phi| \geq l_{0}
\end{array}
$$

where $s_{0}$ is the length of the straight line segment and $r_{0}$ is the radius of curvature of the bends, c.f. $[19,23]$.

The lumped coupling coefficient of the two modes at zero mismatch can then be expressed as

$$
\kappa_{x, y}=\hat{\kappa}_{x, y}\left(s_{0}\right)\left(2 l_{0}+\sqrt{\frac{\pi r_{0}}{\alpha_{x}}}\right) .
$$

\section{A. $Q$-value}

The quality factor $Q$ is commonly used as a figure of merit for the frequency selectivity of a resonator. It is defined as the ratio of energy stored to energy lost per cycle. There are several factors that contribute to the $Q$-value, but the most important, from a design perspective, is the radiation loss, which accounts for coupling in and out of the resonator. This coupling $Q$ can be approximated as [19]

$$
Q_{r} \approx \frac{2 \pi^{2} n_{\mathrm{eff}} r_{0}}{\kappa^{2} \lambda_{0}}
$$

It is also important to take into account the intrinsic absorption loss $\hat{\alpha}$ of the resonator, which gives the contribution

$$
Q_{i} \approx \frac{2 \pi n_{\mathrm{eff}}}{\hat{\alpha} \lambda_{0}}
$$

The total $Q$ can then be found as $Q^{-1}=Q_{r}^{-1}+Q_{i}^{-1}$.

The critical coupling wavelength, which separates the overand under-coupled regime, is found when the coupling coefficient has the same magnitude as the absorption coefficient. A ring resonator coupled to a single waveguide at a resonant wavelength where this condition is satisfied will be able to completely extract the field on resonance, and will have a unity extinction ratio.

Although bending losses can generally be neglected for resonators with a high refractive index contrast, it may, however, be important to take into account the scattering losses from sidewall imperfections. A detailed analysis of these phenomena is beyond the scope of the current paper, but it should be 
noted that such sidewall scattering can also lead to the coupling of appreciable amounts of energy into the counterpropagating mode, which may seriously deteriorate device performance [36].

\section{CONCLUSIONS}

We have presented an analytical approach for obtaining the linear and nonlinear design parameters of planar microring resonators. Approximate analytical solutions of the eigenmode/eigenfrequency problem, using the effective index method for resonators with both a cylindrical and rectangular cross section geometry, have been derived. In the latter case, we have also presented an explicit analytical approximation for the effective and auxiliary refractive indices. Additionally, we have demonstrated how the dispersion profile can be obtained from knowledge of the resonance frequencies and considered the mode dependence of the nonlinear Kerr coefficient by deriving an analytical approximation for the mode area. Finally, we have presented expressions for the coupling coefficients and made estimates of the quality factor. The analytical theory has been compared with good agreement to numerical mode solver simulations, with examples given for microring resonators made of silicon.

The analytical theory presented here should be helpful for designing microresonators that can be used for nonlinear FWM applications. The theory allows one to make rapid approximations of both linear and nonlinear design parameters. The theory is able to accurately estimate the FSR, $Q$-value, and coupling regimes, as well as cavity dispersion properties and mode volumes. This allows analytical estimates to be found for all parameters that appear in the dynamic theories that describe frequency comb generation.

The analytical approach has the advantage over numerical simulations of being both faster and providing additional insight. However, we emphasize that it should be complemented with more precise numerical methods to verify sensitive parameters, such as zero-dispersion wavelengths.

The theory could also be used to obtain estimates and corrections for the higher-order effects that can be of importance for wideband frequency comb generation. Such effects include both thermal and nonlinear mode pulling, which may change the dispersion properties as well as the frequency dependence of nonlinear Kerr and linear coupling coefficients.

\section{ACKNOWLEDGMENTS}

This research was funded by Fondazione Cariplo (grant no. 2011-0395) and the Italian Ministry of University and Research (grant no. 2012BFNWZ2).

\section{REFERENCES}

1. P. Del'Haye, A. Schliesser, O. Arcizet, T. Wilken, R. Holzwarth, and T. J. Kippenberg, "Optical frequency comb generation from a monolithic microresonator," Nature 450, 1214-1217 (2007)

2. F. Ferdous, H. Miao, D. E. Leaird, K. Srinivasan, J. Wang, L. Chen, L. T. Varghese, and A. M. Weiner, "Spectral line-by-line pulse shaping of on-chip microresonator frequency combs," Nat. Photonics 5, 770-776 (2011).

3. A. Schliesser, N. Picqué, and T. W. Hänsch, "Mid-infrared frequency combs," Nat. Photonics 6, 440-449 (2012).

4. R. Holzwarth, T. Udem, T. W. Hänsch, J. C. Knight, W. J. Wadsworth, and P. St. J. Russell, "Optical frequency synthesizer for precision spectroscopy,” Phys. Rev. Lett. 85, 2264-2267 (2000).

5. T. Udem, R. Holzwarth, and T. W. Hänsch, "Optical frequency metrology," Nature 416, 233-237 (2002).

6. Y. Okawachi, K. Saha, J. S. Levy, Y. H. Wen, M. Lipson, and A. L. Gaeta, "Octave-spanning frequency comb generation in a silicon nitride chip," Opt. Lett. 36, 3398-3400 (2011).

7. S. Azzini, D. Grassani, M. Galli, L. C. Andreani, M. Sorel, M. J. Strain, L. G. Helt, J. E. Sipe, M. Liscidini, and D. Bajoni, "From classical four-wave mixing to parametric fluorescence in silicon microring resonators," Opt. Lett. 37, 3807-3809 (2012).

8. A. Biberman, M. J. Shaw, E. Timurdogan, J. B. Wright, and M. R. Watts, "Ultralow-loss silicon ring resonators," Opt. Lett. 37, 4236-4238 (2012).

9. D. K. Armani, T. J. Kippenberg, S. M. Spillane, and K. J. Vahala, "Ultra-high-Q toroid microcavity on a chip," Nature 421, 925-928 (2003).

10. M. Hossein-Zadeh and K. J. Vahala, "Free ultra-high-Q microtoroid: a tool for designing photonic devices," Opt. Express 15, 166-175 (2007).

11. H. Tavernier, P. Salzenstein, K. Volyanskiy, Y. K. Chembo, and L Larger, "Magnesium fluoride whispering gallery mode diskresonators for microwave photonics applications," IEEE Photon. Technol. Lett. 22, 1629-1631 (2010).

12. M. Ferrera, D. Duchesne, L. Razzari, M. Peccianti, R. Morandotti, P. Cheben, S. Janz, D. X. Xu, B. E. Little, S. Chu, and D. J. Moss, "Low power four-wave mixing in an integrated, micro-ring resonator with $Q=1.2$ million," Opt. Express 17, 14098-14103 (2009).

13. B. E. Little, J. S. Foresi, G. Steinmeyer, E. R. Thoen, S. T. Chu H. A. Haus, E. P. Ippen, L. C. Kimerling, and W. Greene, "Ultracompact $\mathrm{Si}-\mathrm{SiO}_{2}$ microring resonator optical channel dropping filters," IEEE Photon. Technol. Lett. 10, 549-551 (1998).

14. A. R. Johnson, Y. Okawachi, J. S. Levy, J. Cardenas, K. Saha, M. Lipson, and A. L. Gaeta, "Chip-based frequency combs with sub$100 \mathrm{GHz}$ repetition rates," Opt. Lett. 37, 875-877 (2012).

15. C. Y. Wang, T. Herr, P. Del'Haye, A. Schliesser, J. Hofer, R. Holzwarth, T. W. Hänsch, N. Picqué, and T. J. Kippenberg, "Mid-infrared optical frequency combs at $2.5 \mu \mathrm{m}$ based on crystalline microresonators," Nat. Commun. 4, 1345 (2013).

16. T. Hansson, D. Modotto, and S. Wabnitz, "Dynamics of the modulational instability in microresonator frequency combs," Phys. Rev. A 88, 023819 (2013).

17. A. B. Matsko and V. S. Ilchenko, "Optical resonators with whispering-gallery modes-part I: basics,” IEEE J. Sel. Top. Quantum Electron. 12, 3-14 (2006).

18. M. L. Gorodetsky and A. E. Fomin, "Geometrical theory of whispering-gallery modes,” IEEE J. Sel. Top. Quantum Electron. 12, 33-39, (2006).

19. B. E. Little, S. T. Chu, H. A. Haus, J. Foresi, and J. P. Laine, "Microring resonator channel dropping filters," J. Lightwave Technol. 15, 998-1005 (1997).

20. M. K. Chin and S. T. Ho, "Design and modeling of waveguidecoupled single-mode microring resonators," J. Lightwave Technol. 16, 1433-1446 (1998).

21. C. Manolatou, M. J. Khan, S. Fan, P. R. Villeneuve, H. A. Haus, and J. D. Joannopoulos, "Coupling of modes analysis of resonant channel add-drop filters," IEEE J. Quantum Electron. 35, 1322-1331 (1999).

22. O. Schwelb, "Transmission, group delay, and dispersion in single-ring optical resonators and add/drop filters - a tutorial overview," J. Lightwave Technol. 22, 1380-1394 (2004).

23. S. J. Emelett and R. Soref, "Design and simulation of silicon microring optical routing switches," J. Lightwave Technol. 23, 1800-1807 (2005).

24. A. B. Matsko, A. A. Savchenkov, W. Liang, V. S. Ilchenko, D. Seidel, and L. Maleki, "Mode-locked Kerr frequency combs," Opt. Lett. 36, 2845-2847 (2011).

25. S. Coen, H. G. Randle, T. Sylvestre, and M. Erkintalo, "Modeling of octave-spanning Kerr frequency combs using a generalized mean-field Lugiato-Lefever model," Opt. Lett. 38, 37-39 (2013).

26. A. Matsko, A. Savchenkov, D. Strekalov, V. Ilchenko, and L. Maleki, "Optical hyperparametric oscillations in a whisperinggallery-mode resonator: threshold and phase diffusion," Phys. Rev. A 71, 033804 (2005) 
27. Y. K. Chembo and N. Yu, "Modal expansion approach to opticalfrequency-comb generation with monolithic whispering-gallerymode resonators," Phys. Rev. A 82, 033801 (2010).

28. M. Sumetsky, "Whispering-gallery-bottle microcavities: the three-dimensional etalon," Opt. Lett. 29, 8-10 (2004).

29 . E. A. J. Marcatili, "Bends in optical dielectric guides," AT\&T Tech. J. 48, 2103-2132 (1969).

30. K. Okamoto, Fundamentals of Optical Waveguides, 2nd ed., (Academic, 2006).

31. E. D. Palik, ed., Handbook of Optical Constants of Solids (Academic, 1998).

32. M. Sumetsky, Y. Dulashko, and R. S. Windeler, "Super free spectral range tunable optical microbubble resonator," Opt. Lett. 35, 1866-1868, (2010).
33. M. Oxborrow, "Traceable 2-D finite-element simulation of the whispering-gallery modes of axisymmetric electromagnetic resonators," IEEE Trans. Microwave Theor. Tech. 55, 1209-1218 (2007).

34. H. A. Haus, W. P. Huang, S. Kawakami, and N. A. Whitaker, "Coupled-mode theory of optical waveguides," J. Lightwave Technol. 5, 16-23 (1987).

35. M. Kuznetsov, "Expressions for the coupling coefficient of a rectangular-waveguide directional coupler," Opt. Lett. 8, 499-501 (1983)

36. F. Morichetti, A. Canciamilla, C. Ferrari, M. Torregiani, A. Melloni, and M. Martinelli, "Roughness induced backscattering in optical silicon waveguides," Phys. Rev. Lett. 104, 033902 (2010) 